\title{
JAIME E. MUÑOZ RIVERA Smoothness effect and decay on a class of non linear evolution equation
}

\author{
Annales de la faculté des sciences de Toulouse $\sigma^{e}$ série, tome $1, \mathrm{n}^{\circ} 2$ \\ (1992), p. 237-260 \\ <http://www.numdam.org/item?id=AFST_1992_6_1_2_237_0>
}

(C) Université Paul Sabatier, 1992, tous droits réservés.

L'accès aux archives de la revue «Annales de la faculté des sciences de Toulouse » (http://picard.ups-tlse.fr/ annales/) implique l'accord avec les conditions générales d'utilisation (http://www.numdam.org/conditions). Toute utilisation commerciale ou impression systématique est constitutive d'une infraction pénale. Toute copie ou impression de ce fichier doit contenir la présente mention de copyright.

\section{Numdam}

Article numérisé dans le cadre du programme

Numérisation de documents anciens mathématiques

http://www.numdam.org/ 


\title{
Smoothness effect and decay on a class of non linear evolution equation
}

\author{
JAIME E. MUÑoz RIVERA ${ }^{(1)}$
}

RÉSUMÉ. - Nous nous proposons dans ce travail de prouver l'existence de solutions du système $u_{t t}+M\left(\left\|A^{1 / 2} u(t)\right\|_{\mid}^{2}\right) A u+A u_{t}=0$, avec les conditions initiales $u(0)=u_{0} \in D(A), u_{t}(0)=u_{1} \in H$, qui décroissent uniformément quand $t \rightarrow+\infty$ et ont la propriété de régularisation des conditions initiales, c'est-à-dire si $u_{0} \in D(A)$ et $u_{1} \in H$ alors la solution du système satisfait $\left.\left.u \in C^{2}(] 0, T\right] ; D\left(A^{k}\right)\right) \forall k \in \mathbb{N}$.

Abstract. - Our aim in this paper is to prove the existence of solution for system $u_{t t}+M\left(\left\|A^{1 / 2} u(t)\right\|^{2}\right) A u+A u_{t}=0$, with initial data $u(0)=$ $u_{0}, u_{t}(0)=u_{1}$ in $D(A)$ and $H$ respectively, which decay uniformly as time goes to infinity and has the smoothness effect property on the initial data $u_{0}$ and $u_{1}$, that is if $u_{0}$ and $u_{1}$ belong to $D(A)$ and $H$ respectively then the corresponding solution satisfies $\left.\left.u \in C^{2}(] 0, T\right] ; D\left(A^{k}\right)\right) \forall k \in \mathbb{N}$.

KEY-WORDS : Existence, smoothness effect, asymptotic behaviour.

\section{Introduction}

Several authors have studied the existence of solutions for the semilinear wave equation given by

$$
u_{t t}-M\left(\|\nabla u(t)\|^{2}\right) \Delta u=0, \quad u(0)=u_{0}, u_{t}(0)=u_{1}
$$

but until now is not proved the existence of global solution when the initial data is taken in the usual Sobolev's spaces, in this direction all the literature

(1) National Laboratory for Scientific Computation, Rua Lauro Muller 455 (LNCCCNPQ), Botafogo, CEP. 22290, Rio de Janeiro R.J. Brasil and IM-UFRJ, P.O. Box 68530, Rio de Janeiro, R.J. Brasil 
concerning this equation is about the existence of local solution. Moreover when the initial data $u_{0}$ and $u_{1}$ are taken in $H_{0}^{1}(\Omega)$ and $L^{2}(\Omega)$ respectively it is not known the existence even for local weak solutions. The existence of global solutions is proved only for analytical initial data, see for example the work of Berstein [2], Pohozaev [6], and Arosio \& Spagnolo [1].

In order to obtain global solution for a class of equations relative to (1.1), several authors ([7], [8], [9] to name but a few) have considered damping terms as $A^{2} u, A u_{t}$ or $A^{\alpha} u_{t}$, which gives strong estimates resulting in the convergence of the nonlinear term, showing in this way global existence results. In Nishihara [9], for example, the author studies the equation

$$
u_{t t}+M\left(\left\|A^{1 / 2} u(t)\right\|^{2}\right) A u+A u_{t}=0, u(0)=u_{0}, u_{t}(0)=u_{1}
$$

and proved the existence and uniqueness of global solution satisfying

$$
\begin{gathered}
u \in C^{1}([0, \infty[; H) \\
u \in L^{\infty}([0, \infty[; D(A)) \\
u_{t} \in L^{\infty}\left(\left[0, \infty\left[; D\left(A^{1 / 2}\right)\right) \cap L^{2}([0, \infty[; D(A))\right.\right. \\
u_{t t} \in L^{2}([0, \infty[, H)
\end{gathered}
$$

when $H$ is a Hilbert space and the following hypotheses hold.

$\mathrm{H} 1 .-A$ is a positive selfadjoint operator of $H$ such that $D(A)$ has compact imbedding in $H$.

H2.- $M$ is c nonnegative $C^{1}$-function.

$\mathrm{H} 3 .-u_{0}$ and $u_{1}$ are given in $D(A)$ and $D\left(A^{1 / 2}\right)$ respectively.

Other weaker dissipative mechanism was studied by Ikehata and Okazawa [3], the authors introduce the friccional damping term $u_{t}$ and show, by using the Yosida's aproximation method together with compactness arguments, the existence of global solution provided the initial data is small enough. This case is different to others studied in [7], [8] and [9] because the compactness method is not enough to obtain global solutions for large data when considered the friccional damping $u_{t}$.

Concerning the asymptotic behaviour Nishihara [9] proves when $M(s)=$ $s$ and $u_{0}$ and $u_{1}$ are given in $D\left(A^{3 / 2}\right)$, that the solution $u(t)$ satisfies the 
following decay properties.

$$
\begin{aligned}
\left\|A^{1 / 2} u(t)\right\|^{2} & \leq C_{\epsilon}(1+t)^{-1+\epsilon}, & \|A u(t)\|^{2} \leq C_{\epsilon}(1+t)^{-\frac{1}{2}+\epsilon} \\
\left\|A^{1 / 2} u_{t}(t)\right\|^{2} & \leq C_{\epsilon}(1+t)^{-3+\epsilon}, & \left\|u_{t t}(t)\right\|^{2} \leq C_{\epsilon}(1+t)^{-4+\epsilon}
\end{aligned}
$$

where $C_{\epsilon} \rightarrow+\infty$ when $\epsilon \rightarrow 0$.

This rate of decay was improved in Matos and Pereira [6], by removing $\epsilon$ in the above inequalities. It is not possible to prove the exponential decay (as is expected when hypothesis $\mathrm{H} 1$ holds) because $M(0)=0$, that is, the equation is degenerate. In this direction Nishihara [10] shows that the rate obtained in [6] is optimal, that is

$$
\left\|A^{1 / 2} u(t)\right\|^{2} \geq C(1+t)^{-1}, \quad \forall t \geq 0 .
$$

On the other hand, when $M(s) \geq m_{0}>0$, Nishihara [9] pointed out that the exponential decay holds, latter Zuazua [12] proved the exponential decay for a general class of hyperbolic equations with damping terms.

To the best of my knowledge there is no result concerning the smoothness effect for equation (1.2).

In this paper we will discuss about some properties which the damping term $A u_{t}$ produces on the solution of equation (1.2), such as global existence, regularity, decay and also the smoothness effect property on the initial data, this means that the solution of (1.2) satisfies $u \in C^{2}\left(\left[0, \infty\left[; D\left(A^{\infty}\right)\right)\right.\right.$ where $D\left(A^{\infty}\right)=\bigcap_{k \in \mathbb{N}} D\left(A^{k}\right)$, no matter the regularity of the initial data have, whenever they are taken in a suitable space (in $D(A) \times H$ for example) in order to obtain the existence result. Smoothness effect is not expected for any non negative continuous function $M$; in fact if $u_{0}$ is such that $M\left(\left\|A^{1 / 2} u_{0}\right\|^{2}\right)=0$, then the function $t \mapsto u(t)=u_{0}$ is the solution of (1.2) when $u_{1}=0$, therefore we have no smoothness effect in this case.

In section 2 we will prove that the smoothness effect holds for any non negative continuous function satisfying $M\left(\left\|A^{1 / 2} u_{0}\right\|^{2}\right)>0$. Existence as well as regularity result are also proved in this section.

Finally in section 3 we will prove that the solution of equation (1.2) decays uniformly as time goes to infinity. 


\section{Existence and regularity}

Let $M:[0,+\infty[\rightarrow[0,+\infty[$ be a non negative continuous function and $A$ satisfying H1. By a weak solution of system (1.2) we mean a function

$$
u:\left[0, \infty\left[\rightarrow D\left(A^{1 / 2}\right)\right.\right.
$$

satisfying

$$
\begin{aligned}
\frac{\mathrm{d}}{\mathrm{d} t}\left(u_{t}, w\right) & +M\left(\left\|A^{1 / 2} u(t)\right\|^{2}\right)\left(A^{1 / 2} u, A^{1 / 2} w\right)+ \\
& +\left(A^{1 / 2} u_{t}, A^{1 / 2} w\right)=0 \\
u(0) & =u_{0} \text { in } D(A), \quad u_{t}(0)=u_{1} \text { in } H,
\end{aligned}
$$

for any $w$ in $D\left(A^{1 / 2}\right)$.

It is well known that there exists sequences $\left(w_{i}\right)_{i \in \mathbb{N}}$ and $\left(\lambda_{i}\right)_{i \in \mathbb{N}}$ of eigenfunctions and eigenvalues of $A$ such that $\left\{w_{i} ; i \in \mathbb{N}\right\}$ is an orthonormal set and $\lambda_{1}<\lambda_{2} \leq \cdots \leq \lambda_{i} \rightarrow \infty$ as $i \rightarrow \infty$. Let us denote by

$$
u_{0}^{(m)}=\sum_{i=1}^{m}\left(u_{0}, w_{i}\right) w_{i} \quad \text { and } \quad u_{1}^{(m)}=\sum_{i=1}^{m}\left(u_{1}, w_{i}\right) w_{i} .
$$

If $u_{0}$ and $u_{1}$ belongs to $D(A)$ and $H$ respectively we have that the following convergence holds

$$
\left(u_{0}^{(m)}, u_{1}^{(m)}\right) \rightarrow\left(u_{0}, u_{1}\right) \text { in } D(A) \times H .
$$

Finally by $V_{m}$ we will denote the space generated by the first $m$ eigenvectors. In this conditions there exists a local solution $u^{(m)}(t)$ on some interval $\left[0, T_{m}[\right.$, for the ordinary differential equation given by

$$
\begin{gathered}
\left(u_{t t}^{(m)}, w\right)+M\left(\left\|A^{1 / 2} u^{(m)}(t)\right\|^{2}\right)\left(A u^{(m)}, w\right)+\left(A u_{t}^{(m)}, w\right)=0 \\
u^{(m)}(0)=u_{0}^{(m)}, \quad u_{t}^{(m)}(0)=u_{1}^{(m)}
\end{gathered}
$$

for any $w$ and $V_{m}$ and $u^{(m)}(t)=\sum_{i=1}^{m} g_{i, m}(t) w_{i}$.

Moreover if $M$ is locally Lipschitz this solution is unique. 
TheOREM 2.1 (Existence). - Let $M$ be a non negative locally Lipschitz function. If $u_{0} \in D(A)$ and $u_{1} \in H$ then there exist a unique global weak solution of (1.2) in the sence of (2.1) satisfying

$$
u \in C([0, \infty] ; D(A)), \quad u_{t} \in C([0, \infty] ; H) \cap L^{2}\left([0, \infty] ; D\left(A^{1 / 2}\right)\right) .
$$

Moreover the identity

$$
E(t)+\int_{0}^{t}\left\|A^{1 / 2} u_{t}(s)\right\|^{2} \mathrm{~d} s=E(0)
$$

holds for any $t \geq \mathbf{0}$, where $E$ is given by

$$
E(t)=\frac{1}{2}\left\|u_{t}(t)\right\|^{2}+\frac{1}{2} \widetilde{M}\left(\left\|A^{1 / 2} u(t)\right\|^{2}\right)
$$

and

$$
\widetilde{M}(s)=\int_{0}^{s} M(\xi) \mathrm{d} \xi
$$

Proof. - In order to facilitate our analysis we will denote by

$$
E_{m}(t)=\frac{1}{2}\left\|u_{t}^{(m)}(t)\right\|^{2}+\frac{1}{2} \widetilde{M}\left(\left\|A^{1 / 2} u^{(m)}(t)\right\|^{2}\right) .
$$

Taking $w=u_{t}^{(m)}$ in (2.2) and integrating the expresion result we have

$$
E_{m}(t)+\int_{0}^{t}\left\|A^{1 / 2} u_{t}^{(m)}(s)\right\|^{2} \mathrm{~d} s=E_{m}(0)
$$

for $t \in\left[0, T_{m}\left[\right.\right.$. Taking $u_{t}^{(m)}$ instead of $w$ in (2.2) we obtain

$$
\left(u_{t t}^{(m)}, u^{(m)}\right)-M\left(\left\|A^{1 / 2} u^{(m)}\right\|^{2}\right)\left(A u^{(m)}, u^{(m)}\right)+\frac{1}{2} \frac{\mathrm{d}}{\mathrm{d} t}\left\|A^{1 / 2} u^{(m)}\right\|^{2}=0 .
$$

Substitution of

$$
\left(u_{t t}^{(m)}(t), u^{(m)}(t)\right)=\frac{\mathrm{d}}{\mathrm{d} t}\left(u_{t}^{(m)}(t), u^{(m)}(t)\right)-\left\|u_{t}^{(m)}(t)\right\|^{2}
$$

in (2.6) yields

$$
\begin{aligned}
& \frac{\mathrm{d}}{\mathrm{d} t}\{\left.\left(u_{t}^{(m)}(t), u^{(m)}(t)\right)+\frac{1}{2}\left\|A^{1 / 2} u^{(m)}(t)\right\|^{2}\right\}= \\
& \quad=\left\|u_{t}^{(m)}(t)\right\|^{2}-M\left(\left\|A^{1 / 2} u^{(m)}(t)\right\|^{2}\right)\left\|A^{1 / 2} u^{(m)}(t)\right\|^{2} \leq \\
& \quad \leq\left\|u_{t}^{(m)}(t)\right\|^{2}
\end{aligned}
$$


for $t \in\left[0, T_{m}[\right.$. From relation $(2.7)$ we obtain

$$
\begin{aligned}
\frac{1}{2}\left\|A^{1 / 2} u^{(m)}(t)\right\|^{2} \leq & \left(u_{1}^{(m)}, u_{0}^{(m)}\right)+\frac{1}{2}\left\|A^{1 / 2} u_{0}^{(m)}\right\|^{2}+ \\
& -\left(u_{t}^{(m)}(t), u^{(m)}(t)\right)+\frac{1}{\lambda_{1}} \int_{0}^{t}\left\|A^{1 / 2} u_{t}^{(m)}(s)\right\|^{2} \mathrm{~d} s,
\end{aligned}
$$

so we have

$$
\begin{aligned}
\frac{1}{2}\left\|A^{1 / 2} u^{(m)}(t)\right\|^{2} \leq & \left(u_{1}^{(m)}, u_{0}^{(m)}\right)+\frac{1}{2}\left\|A^{1 / 2} u_{0}^{(m)}\right\|^{2}+ \\
& +\lambda_{1}^{-1 / 2}\left\|u_{t}^{(m)}(t)\right\|\left\|A^{1 / 2} u^{(m)}(t)\right\| \\
& +\frac{1}{\lambda_{1}} \int_{0}^{t}\left\|A^{1 / 2} u_{t}^{(m)}(s)\right\|^{2} \mathrm{~d} s .
\end{aligned}
$$

By using (2.5) in the above inequality we obtain

$$
\frac{1}{4}\left\|A^{1 / 2} u^{(m)}(t)\right\|^{2} \leq\left(u_{1}^{(m)}, u_{0}^{(m)}\right)+\frac{1}{2}\left\|A^{1 / 2} u_{0}^{(m)}\right\|^{2}+\frac{2}{\lambda_{1}} E_{m}(0),
$$

for $t \in\left[0, T_{m}\left[\right.\right.$. Let's take $w=A u^{(m)}$ in (2.2) then it follows

$$
\begin{aligned}
\frac{\mathrm{d}}{\mathrm{d} t}\{ & \left.\left(u_{t}^{(m)}(t), A u^{(m)}(t)\right)+\frac{1}{2}\left\|A u^{(m)}(t)\right\|^{2}\right\}= \\
& =\left\|A^{1 / 2} u_{t}^{(m)}(t)\right\|^{2}-M\left(\left\|A^{1 / 2} u^{(m)}(t)\right\|^{2}\right)\left\|A u^{(m)}(t)\right\|^{2} \leq \\
& \leq\left\|A^{1 / 2} u_{t}^{(m)}(t)\right\|^{2} .
\end{aligned}
$$

Integration in time yields

$$
\begin{aligned}
\frac{1}{2}\left\|A u^{(m)}(t)\right\|^{2} & \leq-\left(u_{t}^{(m)}(t), A u^{(m)}(t)\right) \times \\
& \times \int_{0}^{t}\left\|A^{1 / 2} u_{t}^{(m)}(\tau)\right\|^{2} \mathrm{~d} \tau+\left(u_{1}^{(m)}, A u_{0}^{(m)}\right)+\frac{1}{2}\left\|A u_{0}^{(m)}\right\|^{2}
\end{aligned}
$$

which implies that

$$
\begin{array}{r}
\frac{1}{4}\left\|A u^{(m)}(t)\right\|^{2} \leq \| \\
u_{t}^{(m)}(t)\left\|^{2}+\int_{0}^{t}\right\| A^{1 / 2} u_{t}^{(m)}(\sigma) \|^{2} \mathrm{~d} \sigma+ \\
+\left(u_{1}^{(m)}, A u_{0}^{(m)}\right)+\frac{1}{2}\left\|A u_{0}^{(m)}\right\|^{2} .
\end{array}
$$


From (2.5) and (2.9) we conclude that the interval $\left[0, T_{m}[\right.$ can be extended to the whole interval $[0,+\infty[$. Moreover we have that

$$
\begin{aligned}
& u_{t}^{(m)} \text { is bounded in } L^{2}\left([0, T] ; D\left(A^{1 / 2}\right)\right) \\
& u^{(m)} \text { is bounded in } L^{\infty}([0, T] ; D(A)) .
\end{aligned}
$$

For any $T>0$. From Lions-Aubin's theorem (see [4] p. 58, th 5.1) we conclude that there exists a subsequence (which we still denoting in the same way) satisfying

$$
\begin{gathered}
u^{(m)} \rightarrow u \text { strongly in } L^{2}\left([0, T] ; D\left(A^{1 / 2}\right)\right) \\
\left\|A^{1 / 2} u^{(m)}(t)\right\| \rightarrow\left\|A^{1 / 2} u(t)\right\| \text { a.e. in }[0, T] .
\end{gathered}
$$

Since $t \mapsto\left\|A^{1 / 2} u^{(m)}(t)\right\|$ is bounded in $L^{\infty}([0, T])$, by the last convergence and the Lebesgue's dominated convergence theorem we have that

$$
M\left(\left\|A^{1 / 2} u^{(m)}(t)\right\|^{2}\right) \rightarrow M\left(\left\|A^{1 / 2} u(t)\right\|^{2}\right) \text { strongly in } L^{2}([0, T])
$$

so $M\left(\left\|A^{1 / 2} u^{(m)}(t)\right\|^{2}\right)$ is a Cauchy sequence in $L^{2}([0, T])$ therefore, for any $\epsilon>0$, there exists $N>0$, such that

$$
\begin{gathered}
\int_{0}^{T}\left|M\left(\left\|A^{1 / 2} u^{(m)}(\sigma)\right\|^{2}\right)-M\left(\left\|A^{1 / 2} u^{(\mu)}(\sigma)\right\|^{2}\right)\right|^{2} \mathrm{~d} \sigma \leq \epsilon, \\
\forall m, \mu \geq N .
\end{gathered}
$$

Our next goal is to prove that $u^{(m)}$ and $u_{t}^{(m)}$ are Cauchy sequences in $C([0, T] ; D(A))$ and $C([0, T] ; H)$ respectively. In order to prove this fact let us take $m$ and $\mu$ any positive natural numbers such that $m>\mu$ and let us define $g_{i, \mu}(t)=0$ for $\mu \leq i \leq m$. In this conditions we can conclude that both $u^{(m)}$ and $u^{(\mu)}$ are aproximation solutions of system (1.2) in $V_{m}$. Let us denote by $U^{(m)}$ the Cauchy difference given by

$$
U^{(m)}=u^{(m)}-u^{(\mu)} .
$$

Since $u_{0}^{(m)}$ and $u_{1}^{(m)}$ are Cauchy sequences in $D(A)$ and $H$ respectively, then there exist $N>0$, such that

$$
\left\|A U^{(m)}(0)\right\|<\epsilon ; \quad\left\|U_{t}^{(m)}(0)\right\|<\epsilon, \quad \forall m, \mu \geq N .
$$


On the other hand, from (2.2) we have that $U^{(m)}$ satisfies

$$
\begin{aligned}
& \left(U_{t t}^{(m)}, w\right)+M\left(\left\|A^{1 / 2} u^{(m)}(t)\right\|^{2}\right)\left(A U^{(m)}, w\right)+\left(A U_{t}^{(m)}, w\right)= \\
& \quad=\left(M\left(\left\|A^{1 / 2} u^{(\mu)}(t)\right\|^{2}\right)-M\left(\left\|A^{1 / 2} u^{(m)}(t)\right\|^{2}\right)\right)\left(A u^{(\mu)}(t), w\right) .
\end{aligned}
$$

Since $u^{(m)}$ is bounded in $H$ so is $U^{(m)}$, taking $w=U_{t}^{(m)}$ in (2.14) and using (2.12) we have

$$
\begin{aligned}
& \frac{1}{2} \frac{\mathrm{d}}{\mathrm{d} t}\left\|U_{t}^{(m)}\right\|^{2}+\frac{1}{2}\left\|A^{1 / 2} U_{t}^{(m)}\right\|^{2} \leq \\
& \leq M\left(\left\|A^{1 / 2} u^{(m)}(t)\right\|^{2}\right)\left(A^{1 / 2} U^{(m)}, A^{1 / 2} U_{t}^{(m)}\right)+ \\
&+\epsilon\left(A^{1 / 2} u^{(\mu)}(t), A^{1 / 2} U_{t}^{(m)}\right)
\end{aligned}
$$

from where it follows

$$
\begin{aligned}
& \frac{1}{2} \frac{\mathrm{d}}{\mathrm{d} t}\left\|U_{t}^{(m)}\right\|^{2}+\frac{1}{4}\left\|A^{1 / 2} U_{t}^{(m)}\right\|^{2} \leq \\
& \quad \leq 4 M\left(\left\|A^{1 / 2} u^{(m)}(t)\right\|^{2}\right)^{2}\left\|A^{1 / 2} U^{(m)}\right\|^{2}+4 \epsilon^{2}\left\|A^{1 / 2} u^{(\mu)}(t)\right\|^{2} .
\end{aligned}
$$

Integrating the above inequality and applying the relation

$$
\|w(t)\|^{2} \leq 2\|w(0)\|^{2}+2 t \int_{0}^{t}\left\|w_{t}(\sigma)\right\|^{2} \mathrm{~d} \sigma
$$

for $A^{1 / 2} U^{(m)}$ instead of $w$, we obtain

$$
\begin{aligned}
\frac{1}{2}\left\|U_{t}^{(m)}\right\|^{2}+\frac{1}{4} \int_{0}^{t}\left\|A^{1 / 2} U_{t}^{(m)}\right\|^{2} \leq & \frac{1}{2}\left\|U_{t}^{(m)}(0)\right\|^{2}+\frac{1}{2} C\left\|A^{1 / 2} U_{t}^{(m)}(0)\right\|+ \\
& +\frac{C T}{2} \int_{0}^{t} \int_{0}^{\tau}\left\|A^{1 / 2} U_{t}^{(m)}(\sigma)\right\|^{2} \mathrm{~d} \sigma \mathrm{d} \tau \\
& +4 \epsilon^{2} t\left\|A^{1 / 2} u^{(\mu)}(t)\right\|^{2}
\end{aligned}
$$

For some positive constant $C$ depending only on the initial data. Applying Gronwall's inequality and relation (2.13) we conclude that $u_{t}^{(m)}$ is a Cauchy sequence, then we have

$$
\begin{aligned}
& u_{t}^{(m)} \rightarrow u_{t} \text { strongly in } L^{2}\left([0, T] ; D\left(A^{1 / 2}\right)\right) \\
& u_{t}^{(m)} \rightarrow u_{t} \text { strongly in } C([0, T] ; H) .
\end{aligned}
$$


Taking $w=A U^{(m)}$ in relation (2.14) and applying the same above reasoning we conclude that $u^{(m)}$ is a Cauchy sequence in $C([0, T] ; D(A))$ then

$$
u^{(m)} \rightarrow u \text { strongly in } C([0, T] ; D(A)) .
$$

From the uniform convergence follows that $u$ and $u_{t}$ satisfies the initial conditions therefore $u$ is a weak solution of equation (1.2). By using the same method as in [9] we conclude the uniqueness. Finally since identity (2.5) holds for any $m \in \mathbb{N}$, by the strong convergence of $u^{(m)}$ and $u_{t}^{(m)}$, easily follows that the energy identity (2.4) holds for any positive value of $t$. The proof is now complete.

Remark 2.1. - It is well known that for any $v \in H$ the following properties holds

$$
\begin{gathered}
v=\sum_{i=1}^{\infty}\left(v, w_{i}\right) w_{i} \\
v \in D(A) \Leftrightarrow \sum_{i=1}^{\infty} \lambda_{i}^{2}\left(v, w_{i}\right)^{2}<\infty .
\end{gathered}
$$

Therefore if $u$ denotes the solution of (1.2) in the sence of theorem 2.1 we have

$$
u(t)=\sum_{i=1}^{\infty}\left(u(t), w_{i}\right) w_{i} .
$$

It is not difficult to prove that the convergence of the above series is uniformly on bounded set of $\mathbf{R}_{+}$.

From now on $g_{i}(t)=\left(u(t), w_{i}\right)$ and $g_{i}^{\prime}(t)=\left(u_{t}(t), w_{i}\right)$ where $u$ is the solution of (1.2). Since $u_{1}^{(m)}$ and $u_{0}^{(m)}$ are Cauchy sequences in $H$ and $D(A)$ respectively we conclude that there exists $\sigma>0$ satisfying

$$
\sigma>\left(u_{1}^{(m)}, u_{0}^{(m)}\right)+\frac{1}{2}\left\|A^{1 / 2} u_{0}^{(m)}\right\|^{2}+\frac{2}{\lambda_{1}} E_{m}(0), \quad \forall m \in \mathbb{N} .
$$

In this conditions we will define $M_{0}$ as

$$
M_{0}=\sup \{M(s) ; s \in[0, \sigma]\} .
$$

Let us denote by $H_{i}(t)$ the following function

$$
H_{i}(t)=\frac{1}{2} g_{i}^{\prime}(t)^{2}+2 M_{0} g_{i}^{\prime}(t) g_{i}(t) .
$$


Finally by $u$ we will denote the solution of (1.2) or its restriction over any subinterval of $[0, \infty[$.

We will conclude the smoothness effect property of $u$ by studing the behaviour of the functions $g_{i}$ defined above.

LEMma 2.1. - Under the hypotheses of theorem 2.1 and for any $i \geq I$, where $I$ is such that $\lambda_{I} \geq 16 M_{0}$, the following inequality holds

$$
H_{i}(t) \leq H_{i}(0) e^{-\lambda_{i} t} \quad \text { for any } t \geq 0 .
$$

Proof. - For simplicity we will denote by $a(t)=M\left(\left\|A^{1 / 2} u(t)\right\|^{2}\right)$, then projecting equation (1.2) on $\mathbb{R} w_{i}$ we have

$$
g_{i}^{\prime \prime}(t)+\lambda_{i} a(t) g_{i}(t)+\lambda_{i} g_{i}^{\prime}(t)=0
$$

multiplying equation $(2.16)$ by $g_{i}^{\prime}(t)$ we obtain

$$
\begin{aligned}
\frac{1}{2} \frac{\mathrm{d}}{\mathrm{d} t} g_{i}^{\prime}(t)^{2} & =-\lambda_{i} a(t) g_{i}(t) g_{i}^{\prime}(t)-\lambda_{i} g_{i}^{\prime}(t)^{2} \leq \\
& \leq\left(2 M_{0} \lambda_{i} a(t)\right)^{\frac{1}{2}}\left|g_{i}(t)\left(\frac{1}{2} \lambda_{i}\right)^{\frac{1}{2}} g_{i}^{\prime}(t)\right|-\lambda_{i} g_{i}^{\prime}(t)^{2}
\end{aligned}
$$

From where it follows

$$
\frac{1}{2} \frac{\mathrm{d}}{\mathrm{d} t} g_{i}^{\prime}(t)^{2} \leq M_{0} \lambda_{i} a(t) g_{i}(t)^{2}-\frac{3}{4} \lambda_{i} g_{i}^{\prime}(t)^{2} .
$$

Let us denote by $\phi_{i}(t)=g_{i}(t) g_{i}^{\prime}(t)$. By (2.16) we have that

$$
\frac{\mathrm{d}}{\mathrm{d} t} \phi_{i}(t)=g_{i}^{\prime}(t)^{2} \lambda_{i} a(t) g_{i}(t)^{2}-\lambda_{i} \phi_{i}(t) .
$$

Multiplying equation (2.18) by $2 M_{0}$ and adding the product result to inequality (2.17), for $\lambda_{i} \geq 16 M_{0}$ we have

$$
\frac{\mathrm{d}}{\mathrm{d} t} H_{i}(t) \leq-\frac{1}{8} \lambda_{i} g_{i}^{\prime}(t)^{2}-\lambda_{i} M_{0} a(t) g_{i}(t)^{2}-\lambda_{i} H_{i}(t) .
$$


Smoothness effect and decay on a class of non linear evolution equation

In particular

$$
\frac{\mathrm{d}}{\mathrm{d} t} H_{i}(t) \leq-\lambda_{i} H_{i}(t)
$$

multiplying by this inequality by $e^{-\lambda_{i} t}$ and integrating in time, the result follows.

In order to conclude the regularity result let us introduce the following space

$$
\mathcal{W}(a, b)=\left\{u \in L^{2}\left([a, b] ; D\left(A^{\alpha}\right)\right) \mid u_{t} \in L^{2}\left([a, b] ; D\left(A^{\beta}\right)\right)\right\}
$$

where $\alpha$ and $\beta$ are positive real numbers, this space with the norm

$$
\|w\|_{\mathcal{W}(a, b)}^{2}=\int_{a}^{b}\left\|A^{\alpha} w(\tau)\right\|_{H}^{2} \mathrm{~d} \tau+\int_{a}^{b}\left\|A^{\beta} w_{t}(\tau)\right\|_{H}^{2} \mathrm{~d} \tau
$$

is a Hilbert space (see [5]). The following lemma will play and important role in the sequel

LEMMA 2.2. - With the above notations we have

$$
\mathcal{W}(a, b) \subset C\left([a, b] ; D\left(A^{(\alpha+\beta) / 2}\right)\right)
$$

and

$$
\left\|A^{(\alpha+\beta) / 2} u(t)\right\| \leq C\|u\|_{\mathcal{W}(a, b)}, \quad \forall t \in[a, b] .
$$

Moreover if $\beta>\alpha$ then for any $\epsilon>0$ we have

$$
\mathcal{W}(a, b) \subset C\left([a, b] ; D\left(A^{\beta-\epsilon}\right)\right)
$$

and

$$
\left\|A^{\beta-\epsilon} u(t)\right\| \leq C_{\epsilon}\|u\|_{\mathcal{W}(a, b)}, \quad \forall t \in[a, b]
$$

Proof. - Since there exist an extension operator $\mathcal{P}$ defined in $\mathcal{W}(a, b)$ into $\mathcal{W}(\mathbf{R})$, we can suppose that $a=-\infty$ and $b=+\infty$ (see Lions [5]). Since the space $C_{0}^{\infty}\left(\mathbb{R} ; D\left(A^{\gamma}\right)\right)$ is dense in $\mathcal{W}(\mathbb{R})$ (where $\left.\gamma=\max \{\alpha, \beta\}\right)$ it is sufficient to prove that for any $u$ in $C_{0}^{\infty}\left(\mathbb{R} ; D\left(A^{\gamma}\right)\right)$ relation $(2.20)$ holds. First note that the function $t \mapsto\left(u(t), w_{i}\right)$ belongs to $L^{2}(\mathbb{R})$ for any $i \in \mathbb{N}$, then by using the Fourier transform

$$
\mathcal{F} v(\xi)=\frac{1}{\sqrt{2 \pi}} \int_{\mathbb{R}} v(\tau) e^{i \tau \xi} \mathrm{d} \tau
$$


Jaime E. Munoz Rivera

we have

$$
\begin{aligned}
\left(u(t), w_{i}\right)^{2}=\left|\frac{1}{\sqrt{2 \pi}} \int_{\mathbb{R}} e^{-i t \tau} \mathcal{F}\left\{\left(u(\tau), w_{i}\right)\right\} \mathrm{d} \tau\right|^{2} \leq \\
\leq \frac{1}{\sqrt{2 \pi}}\left|\int_{\mathbb{R}}\left(\lambda_{i}^{2 \alpha}+\tau^{2} \lambda_{i}^{2 \beta}\right)^{-\frac{1}{2}}\left(\lambda_{i}^{2 \alpha}+\tau^{2} \lambda_{i}^{2 \beta}\right)^{\frac{1}{2}} \mathcal{F}\left\{\left(u(\tau), w_{i}\right)\right\} \mathrm{d} \tau\right|^{2} \\
\leq \frac{1}{\sqrt{2 \pi}} \int_{\mathbb{R}}\left(\lambda_{i}^{2 \alpha}+\tau^{2} \lambda_{i}^{2 \beta}\right)^{-1} \mathrm{~d} \tau \times \\
\quad \times\left\{\int_{\mathbb{R}}\left(\lambda_{i}^{2 \alpha}+\tau^{2} \lambda_{i}^{2 \beta}\right)\left|\mathcal{F}\left\{\left(u(\tau), w_{i}\right)\right\}\right|^{2} \mathrm{~d} \tau\right\} \\
\leq \frac{1}{\sqrt{2 \pi}} \int_{\mathbb{R}}\left(\lambda_{i}^{2 \alpha}+\tau^{2} \lambda_{i}^{2 \beta}\right)^{-1} \mathrm{~d} \tau \times \\
\quad \times\left\{\int_{\mathbb{R}} \lambda_{i}^{2 \alpha}\left|\left(u(\tau), w_{i}\right)\right|^{2}+\lambda_{i}^{2 \beta}\left|\left(u_{t}(\tau), w_{i}\right)\right|^{2} \mathrm{~d} \tau\right\}
\end{aligned}
$$

taking $\sigma=\lambda_{i}^{\beta-\alpha} \tau$ we have

$$
\begin{aligned}
\lambda_{i}^{\alpha+\beta}\left(u(t), w_{i}\right)^{2} \leq & \frac{1}{\sqrt{2 \pi}} \int_{\mathbb{R}}\left(1+\sigma^{2}\right)^{-1} \mathrm{~d} \sigma \times \\
& \times\left\{\int_{\mathbb{R}} \lambda_{i}^{2 \alpha}\left|\left(u(\tau), w_{i}\right)\right|^{2}+\int_{\mathbb{R}} \lambda_{i}^{2 \beta}\left|\left(u_{t}(\tau), w_{i}\right)\right|^{2} \mathrm{~d} \tau\right\}
\end{aligned}
$$

summing up from 1 to $m$ letting $m \rightarrow \infty,(2.20)$ follows. Finally let us suppose that $\beta>\alpha$ and $a, b \in \mathbb{R}$ from (2.20) we obtain that

$$
u \in C\left([a, b] ; D\left(A^{(\alpha+\beta) / 2}\right)\right)
$$

since

$$
u_{t} \in L^{2}\left([a, b] ; D\left(A^{\beta}\right)\right) .
$$

Aplication of (2.20) yields

$$
\begin{aligned}
& \left\|A^{\beta+2^{-2}(\alpha-\beta)} u(t)\right\| \leq \\
& \quad \leq C\left\{\int_{a}^{b}\left\|A^{(\alpha+\beta) / 2} u(\tau)\right\|_{H}^{2} \mathrm{~d} \tau+\int_{a}^{b}\left\|A^{\beta} u_{t}(\tau)\right\|_{H}^{2} \mathrm{~d} \tau\right\} \\
& \quad \leq C_{2}\left\{\int_{a}^{b}\left\|A^{\alpha} u(\tau)\right\|_{H}^{2} \mathrm{~d} \tau+\int_{a}^{b}\left\|A^{\beta} u_{t}(\tau)\right\|_{H}^{2} \mathrm{~d} \tau\right\}
\end{aligned}
$$

$-248-$ 
where $C_{2}=C[C(b-a)+1]$. Applying (2.20) for $\beta+2^{-2}(\alpha-\beta)$ instead of $\alpha$ we have

$$
\begin{aligned}
& \left\|A^{\beta+2^{-3}(\alpha-\beta)} u(t)\right\| \leq \\
& \quad \leq C\left\{\int_{a}^{b}\left\|A^{\beta+2^{-2}(\alpha-\beta)} u(\tau)\right\|_{H}^{2} \mathrm{~d} \tau+\int_{a}^{b}\left\|A^{\beta} u_{t}(\tau)\right\|_{H}^{2} \mathrm{~d} \tau\right\} \\
& \quad \leq C_{3}\left\{\int_{a}^{b}\left\|A^{\alpha} u(\tau)\right\|_{H}^{2} \mathrm{~d} \tau+\int_{a}^{b}\left\|A^{\beta} u_{t}(\tau)\right\|_{H}^{2} \mathrm{~d} \tau\right\}
\end{aligned}
$$

where $C_{3}=C\left[C_{2}(b-a)+1\right]$. Repeating this process $n$ times we conclude

$$
\begin{aligned}
& \left\|A^{\beta+2^{-n}(\alpha-\beta)} u(t)\right\| \leq \\
& \quad \leq C_{n}\left\{\int_{a}^{b}\left\|A^{\alpha} u(\tau)\right\|_{H}^{2} \mathrm{~d} \tau+\int_{a}^{b}\left\|A^{\beta} u_{t}(\tau)\right\|_{H}^{2} \mathrm{~d} \tau\right\}
\end{aligned}
$$

where $C_{n}=C\left[C_{n-1}(b-a)+1\right]$. Finally taking $\epsilon>0$, there exist $n$ such that $2^{-n}(\beta-\alpha)<\epsilon$, so we have

$$
\beta+2^{-n}(\alpha-\beta)>\beta-\epsilon,
$$

then the result follows.

Remark 2.2. - Let $I$ be any natural number, and $v_{1}, v_{2}$ in $L^{2}([a, b] ; H)$ and $L^{\infty}([a, b] ; H)$ respectively such that

$$
\sum_{i=I}^{m} \int_{a}^{b} \lambda_{i}^{2 \ell}\left(v_{1}(\tau), w_{i}\right)^{2} \mathrm{~d} \tau+\sum_{i=I}^{m} \lambda_{i}^{2 \ell}\left(v_{2}(t), w_{i}\right)^{2} \leq C, \quad \forall m \geq I
$$

where $C$ is a positive constant which does not depend on $m$. Then it follows that

$$
v_{1} \in L^{2}\left([a, b] ; D\left(A^{\ell}\right)\right) \quad \text { and } \quad v_{2} \in L^{\infty}\left([a, b] ; D\left(A^{\ell}\right)\right) .
$$

In fact since

$$
\begin{aligned}
\sum_{i=1}^{I} \lambda_{i}^{2 \ell}\left\{\int_{a}^{b}\left(v_{1}(\tau), w_{i}\right)^{2} \mathrm{~d} \tau+\left(v_{2}(t), w_{i}\right)^{2}\right\} \leq \\
\leq \lambda_{I}^{2 \ell} \sum_{i=1}^{I}\left\{\int_{a}^{b}\left(v_{1}(\tau), w_{i}\right)^{2} \mathrm{~d} \tau+\left(v_{2}(t), w_{i}\right)^{2}\right\} .
\end{aligned}
$$


By hypoteses on $v_{1}$ and $v_{2}$ the second member of the last inequality is bounded then there exist a positive constant $C_{1}$ which does not depend on $m$ such that

$$
\int_{a}^{b} \sum_{i=1}^{m} \lambda_{i}^{2 \ell}\left(v_{1}(\tau), w_{i}\right)^{2} \mathrm{~d} \tau+\sum_{i=1}^{m} \lambda_{i}^{2 \ell}\left(v_{2}(t), w_{i}\right)^{2} \leq C_{1}, \quad \forall m \in \mathbb{N} .
$$

Letting $m \rightarrow \infty$ and using remark 2.1 our assertion follows.

Corollary 2.1. - Let $M$ be a non negative continuous function and $\ell \geq 1$. If $u_{0} \in D\left(A^{\ell}\right)$ and $u_{1} \in D\left(A^{\ell-1 / 2}\right)$, then the solution of equation (1.2) satisfies

$$
u \in C\left([0,+\infty] ; D\left(A^{\ell}\right)\right) \cap L^{\infty}\left([0,+\infty] ; D\left(A^{\ell}\right)\right)
$$

and

$$
u_{t} \in C\left([\mathbf{0},+\infty] ; D\left(A^{\ell-1 / 2}\right)\right) \cap L^{\infty}\left([\mathbf{0},+\infty] ; D\left(A^{\ell-1 / 2}\right)\right) .
$$

Proof. - Multiplying relation (2.15) by $\lambda_{i}^{2 \ell}$ and integrating in time the product result we obtain

$$
\frac{1}{2} \int_{0}^{t} \lambda_{i}^{2 \ell} g_{i}^{\prime}(\tau)^{2} \mathrm{~d} \tau+M_{0} \lambda_{i}^{2 \ell} g_{i}(t)^{2} \leq M_{0} \lambda_{i}^{2 \ell} g_{i}(0)^{2}+\lambda_{i}^{2 \ell-1} \max \left\{0, H_{i}(0)\right\} .
$$

Let us denote by $I$ the first natural number for which we have

$$
\lambda_{I} \geq 16 M_{0} .
$$

Then summing up the above inequality fomr $I$ to $m$ we have

$$
\begin{array}{r}
\frac{1}{2} \sum_{i=I}^{m} \int_{0}^{t} \lambda_{i}^{2 \ell} g_{i}^{\prime}(\tau)^{2} \mathrm{~d} \tau+\sum_{i=I}^{m} M_{0} \lambda_{i}^{2 \ell} g_{i}(t)^{2} \leq \\
\leq C\left\{\left\|A^{\ell} u_{C}^{(m)}\right\|^{2}+\left\|A^{\ell-1 / 2} u_{1}^{(m)}\right\|^{2}\right\} .
\end{array}
$$

For some positive constant $C$. Since $u, u_{t} \in L^{\infty}([0, T] ; H)$ remark 2.2 implies

$$
u_{t} \in L^{2}\left([0, T] ; D\left(A^{\ell}\right)\right) \quad \text { and } \quad u \in L^{\infty}\left([0, T] ; D\left(A^{\ell}\right)\right) .
$$


By lemma 2.2 we have $u \in C\left([0, T] ; D\left(A^{\ell}\right)\right)$. Finally (1.2) yields $u_{t t} \in L^{2}\left([0, T] ; D\left(A^{\ell-1}\right)\right)$ and since $u_{t} \in L^{2}\left([0, T] ; D\left(A^{\ell}\right)\right)$ from lemma 2.2 the result follows.

In order to prove the smoothness effect we will define the auxiliarity function $J_{i}(t)$ as

$$
J_{i}(t)=\frac{1}{2} g_{i}^{\prime \prime}(t)^{2}+4 M_{0} g_{i}^{\prime \prime}(t) g_{i}^{\prime}(t) .
$$

In this conditions we have

Lemma 2.3. - Under the hypotheses of theorem 2.1 and $M \in C^{1}$, such that $M\left(\left\|A^{1 / 2} u_{0}\right\|\right)>0$, there exist $C>0$ and $\eta>0$ for which the following inequality holds

$$
J_{i}(t)+C H_{i}(t) \leq\left\{J_{i}(0)+C H_{i}(0)\right\} e^{-\lambda_{i} t}, \quad \forall t \in[0, \eta]
$$

for any $i \geq I$, where $\lambda_{I} \geq 32 M_{0}$.

Proof.- Differentiating in time equation (2.16) we obtain

$$
g_{i}^{\prime \prime \prime}+\lambda_{i} a(t) g_{i}^{\prime}+\lambda_{i} g_{i}^{\prime \prime}+\lambda_{i} a^{\prime}(t) g_{i}=0
$$

Multiplying (2.21) by $g_{i}^{\prime \prime}(t)$ we have

$$
\frac{1}{2} \frac{\mathrm{d}}{\mathrm{d} t} g_{i}^{\prime \prime}(t)^{2}=-\lambda_{i} a(t) g_{i}^{\prime}(t) g_{i}^{\prime \prime}(t)-\lambda_{i} g_{i}^{\prime \prime}(t)^{2}-\lambda_{i} a^{\prime}(t) g_{i}(t) g_{i}^{\prime \prime}(t),
$$

but the inequalities

$$
a(t) g_{i}^{\prime}(t) g_{i}^{\prime \prime}(t) \leq 4 a(t)^{2} g_{i}^{\prime}(t)^{2}+\frac{1}{16} g_{i}^{\prime \prime}(t)^{2}
$$

and

$$
a^{\prime}(t) g_{i}(t) g_{i}^{\prime \prime}(t) \leq 4 a^{\prime}(t)^{2} g_{i}(t)^{2}+\frac{1}{16} g_{i}^{\prime \prime}(t)^{2},
$$

holds for any $t>0$; therefore using the fact that $a(t) \leq M_{0}$ we conclude that

$$
\frac{1}{2} \frac{\mathrm{d}}{\mathrm{d} t} g_{i}^{\prime \prime}(t)^{2} \leq 4 M_{0} \lambda_{i} a(t) g_{i}^{\prime}(t)^{2}-\frac{7}{8} \lambda_{i} g_{i}^{\prime \prime}(t)^{2}+4 \lambda_{i} a^{\prime}(t)^{2} g_{i}(t)^{2} .
$$

Let us denote by $\psi_{i}(t)=g_{i}^{\prime}(t) g_{i}^{\prime \prime}(t)$. Differentiating $\psi_{i}$ and using (2.21) we obtain

$$
\frac{\mathrm{d}}{\mathrm{d} t} \psi_{i}(t)=g_{i}^{\prime \prime}(t)^{2}-\lambda_{i} a(t) g_{i}^{\prime}(t)^{2}-\lambda_{i} \psi_{i}(t)-\lambda_{i} a^{\prime}(t) g_{i}(t) g_{i}^{\prime}(t) .
$$


Multiplying equation (2.23) by $4 M_{0}$ and adding up the relation result to inequality $(2.22)$ we obtain

$$
\begin{aligned}
\frac{\mathrm{d}}{\mathrm{d} t} J_{i}(t) \leq & \left\{4 M_{0}-\frac{3}{8} \lambda_{i}\right\} g_{i}^{\prime \prime}(t)^{2}-\lambda_{i} J_{i}(t)+4 \lambda_{i} a^{\prime}(t)^{2} g_{i}(t)^{2}+ \\
& -4 M_{0} \lambda_{i} a^{\prime}(t) g_{i}(t) g_{i}^{\prime}(t)
\end{aligned}
$$

For $\lambda_{i} \geq \frac{32}{3} M_{0}$, we have that

$$
\frac{\mathrm{d}}{\mathrm{d} t} J_{i}(t) \leq-\lambda_{i} J_{i}(t)+4 \lambda_{i}\left(1+16 M_{0}^{2}\right) a^{\prime}(t)^{2} g_{i}(t)^{2}+\frac{1}{16} \lambda_{i} g_{i}^{\prime}(t)^{2} .
$$

From (2.19) we conclude that

$$
\begin{aligned}
\frac{\mathrm{d}}{\mathrm{d} t} & \left\{J_{i}(t)+C H_{i}(t)\right\} \leq \\
\leq & -\lambda_{i} J_{i}(t)-C \lambda_{i} H_{i}(t)+\lambda_{i}\left\{4\left(1+16 M_{0}^{2}\right) a^{\prime}(t)^{2}-C M_{0} a(t)\right\} g_{i}(t)^{2}+ \\
& +\lambda_{i}\left(-\frac{C}{8}+\frac{1}{16}\right) g_{i}^{\prime}(t)^{2} .
\end{aligned}
$$

On the other hand, since $a(0)=M\left(\left\|A^{1 / 2} u_{0}\right\|^{2}\right)>0$ there exist $\eta>0$ for which we have

$$
a(t)>\frac{1}{2} M\left(\left\|A^{1 / 2} u_{0}\right\|^{2}\right), \quad \forall t \in[0, \eta] .
$$

Taking $C=\max \{r, 1\}$ where $r=8\left(1+16 M_{0}^{2}\right)\left\|a^{\prime}\right\|_{\infty}^{2} /\left\{a(0) M_{0}\right\}$ and $\left\|a^{\prime}\right\|_{\infty}=\sup \left\{\left|a^{\prime}(t)\right|^{2} ; t \in[0, T]\right\}$ we have

$$
4\left(1+16 M_{0}^{2}\right) a^{\prime}(t)^{2}-C M_{0} a(t) \leq 0, \quad \forall t \in[0, \eta]
$$

then

$$
\frac{\mathrm{d}}{\mathrm{d} t}\left\{J_{i}(t)+C H_{i}(t)\right\} \leq-\lambda_{i} J_{i}(t)-C \lambda_{i} H_{i}(t), \quad \forall t \in[0, \eta]
$$

multiplying by $e^{-\lambda_{i} t}$ and intregrating in time, the result follows. $\square$ 
Our next goal is to prove that for any $\delta>0$, small enough, the solution $u$ of equation (1.2) satisfies the following property

$$
u(\delta), u_{t}(\delta) \in D\left(A^{\infty}\right),
$$

which together with corollary 2.1 implies that the solution of equation (2.2) with initial datum given by $u(\delta), u_{t}(\delta)$ satisfies

$$
u \in C^{1}\left([\delta, T] ; D\left(A^{\infty}\right)\right)
$$

that implies, by the uniqueness and the arbitrary choose of $\delta$, that the solution $u$ satisfies the smoothness effect property.

LEMMA 2.4. - Under the hypotheses and notations of lemma 2.3 let us suppose that

$$
u \in L^{\infty}\left([\delta, \eta] ; D\left(A^{s}\right)\right)
$$

for $0<\delta<\eta$ and $s$ a positive real number. In this conditions we have

$$
u_{t} \text { and } u_{t t} \in L^{\infty}\left([\delta, \eta] ; D\left(A^{s}\right)\right) \text {. }
$$

Proof. - It is easy to see that

$$
\begin{aligned}
4 M_{0} g_{i}^{\prime \prime}(t) g_{i}^{\prime}(t) & \geq-\frac{1}{4} g_{i}^{\prime \prime}(t)^{2}-16 M_{0}^{2} g_{i}^{\prime}(t)^{2} \\
2 C M_{0} g_{i}^{\prime}(t) g_{i}(t) & \geq-2 C^{2} M_{0}^{2} g_{i}^{\prime}(t)^{2}-\frac{1}{2} g_{i}(t)^{2} .
\end{aligned}
$$

Recalling the definition of $J_{i}$ and $H_{i}$ we have

$$
J_{i}(t)+C H_{i}(t)=\frac{1}{2} g_{i}^{\prime \prime}(t)^{2}+4 M_{0} g_{i}^{\prime \prime}(t) g_{i}^{\prime}(t)+\frac{C}{2} g_{i}^{\prime}(t)^{2}+2 C M_{0} g_{i}^{\prime}(t) g_{i}(t)
$$

therefore

$$
J_{i}(t)+C H_{i}(t) \geq \frac{1}{4} g_{i}^{\prime \prime}(t)^{2}-\left(16+2 C^{2}-\frac{C}{2}\right) M_{0}^{2} g_{i}^{\prime}(t)^{2}-\frac{1}{2} g_{i}(t)^{2} .
$$

From lemma 2.2 and lemma 2.3 we have

$$
\begin{aligned}
\frac{1}{4} g_{i}^{\prime \prime}(t)^{2} \leq & \left(16+2 C^{2}-\frac{C}{2}\right) M_{0}^{2} g_{i}^{\prime}(t)^{2}+ \\
& +\frac{1}{2} g_{i}(t)^{2}+\left\{J_{i}(0)+C H_{i}(0)\right\} e^{-\lambda_{i} t} \\
\frac{1}{4} g_{i}^{\prime}(t)^{2} \leq & 4 M_{0}^{2} g_{i}(t)^{2}+H_{i}(0) e^{-\lambda_{i} t}
\end{aligned}
$$


Denoting by

$$
c_{1}=\left(16+2 C^{2}-\frac{C}{2}\right) M_{0}^{2}
$$

inequalities (2.24) and (2.25) yields

$$
\begin{aligned}
\frac{1}{4} g_{i}^{\prime \prime}(t)^{2} \leq & \left\{16 c_{1} M_{0}^{2}+\frac{1}{2}\right\} g_{i}(t)^{2}+ \\
& +\left\{J_{i}(0)+\left(c_{1}+C\right) H_{i}(0)\right\} e^{-\lambda_{i} t} .
\end{aligned}
$$

On the other hand the hypotheses on $u$ implies that there exist a constant $C$ which does not depend on $m$ satisfying

$$
\sum_{i=I}^{m} \lambda_{i}^{2 s} g_{i}(t)^{2}<C, \quad \forall m \geq I .
$$

Therefore multiplying inequality (2.25) and (2.26) by $\lambda_{i}^{2 s}$, summing up the product result from $I$ to $m$ and using remark 2.2 we obtain

$$
u_{t} \text { and } u_{t t} \in L^{\infty}\left([\delta, \eta] ; D\left(A^{s}\right)\right)
$$

as requerided.

Now we are in condition to prove the main result of this paper.

THEOREM 2.2. - Under the hypotheses of lemma 2.3, the solution $u$ of (1.2) satisfies

$$
u \in C^{2}(] 0,+\infty\left[; D\left(A^{\infty}\right)\right) .
$$

Proof. - We will prove that $u \in C^{2}\left([\delta, T] ; D\left(A^{n}\right)\right)$ for any natural number $n$. In fact since $u \in C([0, T] ; D(A))$, from lemma 2.4 we conclude that

$$
u_{t}, u_{t t} \in L^{\infty}([\delta, \eta] ; D(A))
$$

from where it follows that

$$
u_{t t}=-a(t) A u-A u_{t} \in L^{\infty}([\delta, \eta] ; D(A)) \text { for } \delta \leq t \leq \eta
$$

which is equivalent to

$$
e^{\int_{0}^{t} a(\sigma) \mathrm{d} \sigma} u_{t t}=\frac{\mathrm{d}}{\mathrm{d} t}\left\{e^{\int_{0}^{t} a(\sigma) \mathrm{d} \sigma} A u\right\} \in L^{\infty}([\delta, \eta] ; D(A)) .
$$


Smoothness effect and decay on a class of non linear evolution equation

Denoting by

$$
v=e^{\int_{0}^{t} a(\sigma) \mathrm{d} \sigma} u
$$

we have that

$$
\frac{\mathrm{d} v}{\mathrm{~d} t} \in L^{\infty}([\delta, \eta] ; D(A)) \quad \text { and } \quad \frac{\mathrm{d}}{\mathrm{d} t} A v \in L^{\infty}([\delta, \eta] ; D(A))
$$

therefore it follows that

$$
\frac{\mathrm{d} v}{\mathrm{~d} t} \in L^{\infty}\left([\delta, \eta] ; D\left(A^{2}\right)\right) .
$$

On the other hand since

$$
v \in L^{\infty}([\delta, \eta] ; D(A))
$$

from (2.27), (2.28) and the second part of lemma 2.2 we conclude that

$$
v \in C\left([\delta, \eta] ; D\left(A^{2-2^{-1} \epsilon}\right)\right)
$$

for a small $\epsilon>0$. That is, we have that

$$
u \in C\left([\delta, \eta] ; D\left(A^{2-2^{-1} \epsilon}\right)\right) .
$$

Repeating this reasoning, from lemma 2.4, we obtain that

$$
u_{t}, u_{t t} \in L^{\infty}\left([\delta, \eta] ; D\left(A^{2-2^{-1} \epsilon}\right)\right)
$$

by (1.2) we have that

$$
\frac{\mathrm{d} v}{\mathrm{~d} t} \in L^{\infty}\left([\delta, \eta] ; D\left(A^{2-2^{-1} \epsilon}\right)\right)
$$

and

$$
\frac{\mathrm{d}}{\mathrm{d} t} A v \in L^{\infty}\left([\delta, \eta] ; D\left(A^{2-2^{-1} \epsilon}\right)\right)
$$

therefore we have that

$$
\frac{\mathrm{d} v}{\mathrm{~d} t} \in L^{\infty}\left([\delta, \eta] ; D\left(A^{3-2^{-1} \epsilon}\right)\right) .
$$

From (2.28), (2.29), and the second part of lemma 2.1, we obtain

$$
u \in C\left([\delta, \eta] ; D\left(A^{3-2^{-1} \epsilon-4^{-1} \epsilon}\right)\right) .
$$


Repeating this reasoning $n+1$ times we conclude that

$$
u \in C\left([\delta, \eta] ; D\left(A^{n+2-\rho}\right)\right),
$$

where

$$
\rho=\epsilon \sum_{i=1}^{n+2}\left(\frac{1}{2}\right)^{i} .
$$

By lemma 2.4, we obtain that

$$
u_{t}, u_{t t} \in L^{\infty}\left([\delta, \eta] ; D\left(A^{n+2-\rho}\right)\right),
$$

then by using the first part of lemma 2.1 , we conclude that

$$
u \in C^{1}\left([\delta, \eta] ; D\left(A^{n+1}\right)\right) .
$$

From corollary 2.1 and for the uniqueness of solutions, we conclude that

$$
u \in C^{1}\left(\left[\delta,+\infty\left[; D\left(A^{n+1}\right)\right)\right.\right.
$$

and from (1.2), we obtain that $u \in C^{2}\left(\left[\delta,+\infty\left[; D\left(A^{n}\right)\right)\right.\right.$ as requerided.

\section{Asymtotic Behaviour}

In this section we will prove that the solution of equation (1.2) decay uniformly to zero as time goes to infinity. The method we use here is based on the construction of a Liapunov functional which its derivativee is negative proporcional to a power of itself, therefore an uniformly decay holds.

First of all we will prove the following lemma.

LemMa 3.1. - Let $f$ be a $C^{1}$ function satisfying

$$
\frac{\mathrm{d}}{\mathrm{d} t} f(t) \leq-c f(t)+C(1+t)^{-\beta}
$$

where $c, C$ and $\beta$ are positives constants. Then there exist $T>0$ for which we have

$$
f(t) \leq C(1+t)^{-\beta}, \quad \forall t \geq T .
$$


Proof. - Multiplying the condition on $f$ by $e^{c t}$ and integrating by part the product result yields

$$
e^{c t} f(t) \leq f(0)-\frac{C}{c}+\frac{C}{c} e^{c t}(1+t)^{-\beta}+\beta \frac{C}{c} \int_{0}^{t} e^{c \sigma}(1+\sigma)^{-\beta-1} \mathrm{~d} \sigma .
$$

Since the function $\sigma \mapsto e^{c \sigma}(1+\sigma)^{-\beta-1}$ is non decreasing for $\sigma \geq$ $((\beta+1) / c)-1$ we have that

$$
\begin{aligned}
e^{c t} f(t) & \leq f(0)-\frac{C}{c}+\frac{C}{c} e^{c t}(1+t)^{-\beta}+\beta \frac{C}{c} 2 e^{c \beta}+\beta \frac{C}{c} t e^{c t}(1+t)^{-\beta-1} \\
& \leq f(0)-\frac{C}{c}+(1+\beta) \frac{C}{c} e^{c t}(1+t)^{-\beta}+\beta \frac{C}{c} 2 e^{c \beta} .
\end{aligned}
$$

From this inequality our result follows.

THEOREM 3.1. - Under the hypotheses of theorem 2.1 and $M$ a $C^{1}$-non negative monotonic function satisfying

$$
\liminf _{s \rightarrow 0^{+}} \frac{M(s)}{s^{\alpha}}>0 ; \quad M(s)=O\left(s^{\alpha}\right), \quad s \rightarrow 0 ;
$$

there exist a positive constant $C$ satisfying

$$
\begin{aligned}
\left\|A^{1 / 2} u(t)\right\|^{2} & \leq c(1+t)^{-\frac{1}{\alpha}} \\
\left\|A^{1 / 2} u_{t}(t)\right\|^{2} & \leq C(1+t)^{-2-\frac{1}{\alpha}} \\
\left\|u_{t t}(t)\right\|^{2} & \leq C(1+t)^{-\gamma}
\end{aligned}
$$

where $\gamma=\min \left\{4+\frac{1}{\alpha}, 2+\frac{3}{\alpha}\right\}$.

Proof. - Let us multiply equation (1.2) by $u_{t}$ then we have

$$
\frac{\mathrm{d}}{\mathrm{d} t} E(t)=-\left\|A^{1 / 2} u_{t}(t)\right\|^{2} .
$$

Let us define the auxiliarity function $\rho$ as

$$
\rho(t)=\left(u_{t}(t), u(t)\right)+\frac{1}{2}\left\|A^{1 / 2} u(t)\right\|^{2}
$$

differentiating $\rho$ and using (1.2), we obtain

$$
\frac{\mathrm{d}}{\mathrm{d} t} \rho(t)=\left\|u_{t}(t)\right\|^{2}-M\left(\left\|A^{1 / 2} u(t)\right\|^{2}\right)\left\|A^{1 / 2} u(t)\right\|^{2} .
$$


From (3.4), (3.5) and $\epsilon$ small enough we have

$$
\begin{aligned}
\frac{\mathrm{d}}{\mathrm{d} t}\{E(t)+\epsilon \rho(t)\} & \leq-(1-c \epsilon)\left\|A^{1 / 2} u_{t}(t)\right\|^{2}-\epsilon \widetilde{M}\left(\left\|A^{1 / 2} u(t)\right\|^{2}\right) \\
& \leq-\left[1-\left(c+\frac{1}{2} \lambda_{1}^{-1}\right) \epsilon\right]\left\|A^{1 / 2} u_{t}(t)\right\|^{2}-\epsilon E(t) \\
& \leq-\epsilon E(t) .
\end{aligned}
$$

From this inequality we conclude that $E \in L^{1}(\mathbb{R})$. On the other hand from (3.4) it follows that

$$
\frac{\mathrm{d}}{\mathrm{d} t}\{(1+t) E(t)\} \leq E(t) .
$$

Integration in time implies that there exist a positive constant $C$ satisfying

$$
E(t) \leq C(1+t)^{-1} .
$$

Here $C$ will denote a generic constant which does not depend on $\epsilon$. Since $M$ is a increasing function the last inequality implies that $\left\|A^{1 / 2} u(t)\right\| \rightarrow 0$ as $t \rightarrow \infty$. Then by hypothesis on $M$ we conclude that there exist $N>0$ such that

$$
\left\|A^{1 / 2} u(t)\right\|^{2(\alpha+1)} \leq C \widetilde{M}\left(\left\|A^{1 / 2} u(t)\right\|^{2}\right) \quad \text { for } t \geq N,
$$

then it follows that

$$
[E(t)+\epsilon \rho(t)]^{\alpha+1} \leq C E(t) .
$$

Multiplying (3.6) by $C$ and (3.7) by $\epsilon$ and adding the products results, we have

$$
\frac{\mathrm{d}}{\mathrm{d} t}\{E(t)+\epsilon \rho(t)\}+\frac{C}{\epsilon}\{E(t)+\epsilon \rho(t)\}^{\alpha} \leq 0 .
$$

From where it follows that

$$
E(t)+\epsilon \rho(t) \leq C(1+t)^{-\frac{1}{\alpha}} .
$$

On the other hand taking $\epsilon$ small enough we have that there exist a positive constant $c$ such that

$$
E(t)+\epsilon \rho(t) \geq c\left\{\left\|u_{t}(t)\right\|^{2}+\widetilde{M}\left(\left\|A^{1 / 2} u(t)\right\|^{2}\right)+\left\|A^{1 / 2} u(t)\right\|^{2}\right\} .
$$


From (3.8) and (3.9), (3.1) follows. Since $M \in C^{1}$, we can differentiate equation (1.2) and multiplying by $u_{t t}$ the equation result for $t>0$, we have

$$
\begin{aligned}
& \frac{\mathrm{d}}{\mathrm{d} t}\left\|u_{t t}(t)\right\|^{2}=-\left\|A^{1 / 2} u_{t t}(t)\right\|^{2}+ \\
& \quad-M\left(\left\|A^{1 / 2} u(t)\right\|^{2}\right)\left(A u_{t}(t), u_{t t}(t)\right)+ \\
& \quad-M^{\prime}\left(\left\|A^{1 / 2} u(t)\right\|^{2}\right)\left(u(t), A u_{t}(t)\right)\left(A^{1 / 2} u(t), A^{1 / 2} u_{t t}(t)\right) .
\end{aligned}
$$

Since

$$
A u_{t}(t)=-u_{t t}-M\left(\left\|A^{1 / 2} u(t)\right\|^{2}\right) A u(t)
$$

sustitution in (3.10) yields

$$
\begin{aligned}
& \frac{\mathrm{d}}{\mathrm{d} t}\left\|u_{t t}(t)\right\|^{2}=-\left\|A^{1 / 2} u_{t t}(t)\right\|^{2}+ \\
& +M\left(\left\|A^{1 / 2} u(t)\right\|^{2}\right)\left\|u_{t t}(t)\right\|^{2}+ \\
& +M\left(\left\|A^{1 / 2} u(t)\right\|^{2}\right)^{2}\left(A^{1 / 2} u(t), A^{1 / 2} u_{t t}(t)\right) \times \\
& \quad \times \quad M^{\prime}\left(\left\|A^{1 / 2} u(t)\right\|^{2}\right)\left(u(t), u_{t t}(t)\right)\left(A^{1 / 2} u(t), A^{1 / 2} u_{t t}(t)\right)+ \\
& +M\left(\left\|A^{1 / 2} u(t)\right\|^{2}\right) M^{\prime}\left(\left\|A^{1 / 2} u(t)\right\|^{2}\right)\left\|A^{1 / 2} u(t)\right\|^{2} \times \\
& \quad \times\left(A^{1 / 2} u(t), A^{1 / 2} u_{t t}(t)\right) .
\end{aligned}
$$

Using (3.1) for $t \geq N, N$ big enough, we have

$$
\begin{aligned}
\frac{\mathrm{d}}{\mathrm{d} t}\left\|u_{t t}(t)\right\|^{2}= & -\frac{1}{2}\left\|A^{1 / 2} u_{t t}(t)\right\|^{2}+ \\
& +C M\left(\left\|A^{1 / 2} u(t)\right\|^{2}\right)^{4}\left\|A^{1 / 2} u(t)\right\|^{2}+ \\
& +C M\left(\left\|A^{1 / 2} u(t)\right\|^{2}\right)^{2}\left\|A^{1 / 2} u(t)\right\|^{4} .
\end{aligned}
$$

Using the above decay result, lemma 3.1 and since

$$
M\left(\left\|A^{1 / 2} u(t)\right\|^{2}\right) \leq C(1+t)^{-1}, \quad \forall t \geq N
$$

we obtain (3.3). Finally multiplying (1.2) by $u_{t}$ we have that

$$
\begin{gathered}
\left\|A^{1 / 2} u_{t}(t)\right\|^{2} \leq M\left(\left\|A^{1 / 2} u(t)\right\|^{2}\right)\left\|A^{1 / 2} u_{t}(t)\right\|\left\|A^{1 / 2} u(t)\right\|+ \\
+\left\|u_{t t}(t)\right\|\left\|u_{t}(t)\right\| \\
-259-
\end{gathered}
$$


from where it follows

$$
\begin{aligned}
\frac{1}{2}\left\|A^{1 / 2} u_{t}(t)\right\|^{2} \leq & 4 M\left(\left\|A^{1 / 2} u(t)\right\|^{2}\right)^{2}\left\|A^{1 / 2} u_{t}(t)\right\|^{2}+ \\
& +\frac{4}{\lambda_{1}}\left\|u_{t t}(t)\right\|^{2}
\end{aligned}
$$

using the above decay rates we obtain (3.2). Then our result follows.

\section{References}

[1] Arosio (A.) and SPAGnolo (S.) . - Global solution of the Cauchy problem for a nonlinear hyperbolic equation. Non linear partial diferential equation and their applications,

College de France Seminar, Vol 6. Edited by H. Brezis \& J. L. Lions, PitmanLondon (1984) pp. 1-26.

[2] Berstein (S.). - Sur une classe d'équations fonctionnelles aux dérivées partielles, Izv. Akad. Nauk SSSR, Ser. Mat. 4 (1940) pp. 17-26 (Math. Rev. 2 n $^{\circ} 102$ ).

[3] IKehata (R.) and OKazaWa (N.) . - Yosida approximation and nonlinear hyperbolic equation,

Nonlinear Analysis, Theory, Method and Application, Vol. 15, 5 (1990) pp. 479495.

[4] Lions (J.L.) .- Quelques Méthodes de résolution de problèmes aux limites non linéaires,

Dunod Gauthier Villars, Paris (1969).

[5] Lions (J.L.) and Magenes (E.).- Non Homogeneous Boundary Value Problems and aplications,

Springer-Verlag New-York (1972).

[6] Matos (M.P.) and Pereira (D.C.).- On a nonlinear wave equation with strong damping,

Funkcial Ekvac., 34 (1991) pp. 303-311.

[7] Medeiros (L.A.) and Milla Miranda (M.). - On a non linear wave equation with damping,

Revista de Matematica de la Universidad Complutense de Madrid, Vol. 3, 2 (1990).

[8] Menzala (G.P.) - - On global classical solutions of a non linear wave equation, Applicable analysis, 10 (1980) pp. 179-195.

[9] Nishihara (K.) .- Degenerate quasilinear hyperbolic equation with strong damping,

Funkcialaj Ekvacioj, 27 (1984) pp. 125-145.

[10] Nishihara (K.) . - Decay properties of solutions of some quasilinear hyperbolic equation with strong damping,

To appear.

[11] Pоноzaev (S.I.) .- On a class of quasilinear hyperbolic equation,

Math. USSR-Sb., 25-1 (1975) pp. 145-158.

[12] ZUAZUA (E.) .- Stability and Decay for a class of non linear hyperbolic problems, Asymptotic Analysis 1 (1988) pp. 161-185. 